\title{
Analysis of the potential for a malaria vaccine to reduce gaps in malaria intervention coverage
}

\author{
H. Juliette T. Unwin ${ }^{1}$ (D, Lazaro Mwandigha ${ }^{1,2}$, Peter Winskill ${ }^{1}$, Azra C. Ghani ${ }^{1}$ and Alexandra B. Hogan ${ }^{*}$ (D)
}

\begin{abstract}
Background: The RTS,S/AS01 malaria vaccine is currently being evaluated in a cluster-randomized pilot implementation programme in three African countries. This study seeks to identify whether vaccination could reach additional children who are at risk from malaria but do not currently have access to, or use, core malaria interventions.

Methods: Using data from household surveys, the overlap between malaria intervention coverage and childhood vaccination (diphtheria-tetanus-pertussis dose 3, DTP3) uptake in 20 African countries with at least one first administrative level unit with Plasmodium falciparum parasite prevalence greater than $10 \%$ was calculated. Multilevel logistic regression was used to explore patterns of overlap by demographic and socioeconomic variables. The public health impact of delivering RTS,S/AS01 to those children who do not use an insecticide-treated net (ITN), but who received the DTP3 vaccine, was also estimated.
\end{abstract}

Results: Uptake of DTP3 was higher than malaria intervention coverage in most countries. Overall, 34\% of children did not use ITNs and received DTP3, while 35\% of children used ITNs and received DTP3, although this breakdown varied by country. It was estimated that there are 33 million children in these 20 countries who do not use an ITN. Of these, 23 million (70\%) received the DTP3 vaccine. Vaccinating those 23 million children who receive DTP3 but do not use an ITN could avert up to an estimated 9.7 million (range 8.5-10.8 million) clinical malaria cases each year, assuming all children who receive DTP3 are administered all four RTS,S doses. An additional 10.8 million (9.5-12.0 million) cases could be averted by vaccinating those 24 million children who receive the DTP3 vaccine and use an ITN. Children who had access to or used an ITN were 9-13\% more likely to reside in rural areas compared to those who had neither intervention regardless of vaccination status. Mothers' education status was a strong predictor of intervention uptake and was positively associated with use of ITNs and vaccination uptake and negatively associated with having access to an ITN but not using it. Wealth was also a strong predictor of intervention coverage.

Conclusions: Childhood vaccination to prevent malaria has the potential to reduce inequity in access to existing malaria interventions and could substantially reduce the childhood malaria burden in sub-Saharan Africa, even in regions with lower existing DTP3 coverage.

Keywords: Malaria vaccine, RTS,S/AS01, Expanded Programme on Immunization, Demographic and Health Surveys, DHS Program

\footnotetext{
*Correspondence: a.hogan@imperial.ac.uk

${ }^{1}$ MRC Centre for Global Infectious Disease Analysis, School of Public

Health, Imperial College London, St Mary's Campus, Norfolk Place, London W2 1PG, UK

Full list of author information is available at the end of the article
}

\section{Background}

The introduction of the Millennium Development Goals in 2000 helped to catalyse widespread scale-up of core contemporary malaria control interventions in 
sub-Saharan Africa: insecticide-treated nets (ITNs), indoor residual spraying (IRS), chemoprevention for pregnant women, and more recently, chemoprevention of children in areas of seasonal transmission [1]. Access to treatment of clinical malaria with artemisinin-based combination therapy also increased [1]. Although malaria burden has declined significantly over the previous two decades, progress has recently stalled, with the 10 highest-burden African countries reporting an increase in cases in 2017. Malaria now remains a leading cause of childhood morbidity and mortality. Worldwide in 2019 there were an estimated 229 million cases of malaria and 409,000 deaths, with over $90 \%$ of the deaths occurring in sub-Saharan Africa [2]. Funding for malaria has remained relatively stable since 2010, but the level of investment remains far short of what is required under the World Health Organization (WHO) Global Technical Strategy for Malaria (GTS) [3]. The WHO, alongside other partners, is now prioritizing the country-led "high burden to high impact" initiative, which aims to strategically reduce malaria cases and deaths in the highest burden settings through optimally delivered packages of malaria interventions, coordinated across health and other sectors [4].

Between 2016 and 2018, 578 million ITNs were delivered globally, compared to a total of 582 million between 2014 and 2016 [5, 6]. However, reported usage of ITNs has improved only marginally since 2015 [6]. In addition, fewer people at risk of malaria are being protected by IRS; globally, IRS protection declined from a peak of $5 \%$ in 2010 to $2 \%$ in 2018 [6]. Furthermore, a high proportion of febrile children do not receive medical care (median: 36\%, IQR: $28-45 \%$ based on a separate analysis of 20 household surveys conducted in sub-Saharan Africa between 2015 and 2018) [6]. Uptake of these interventions varies within and between countries and is not evenly distributed across demographic and socioeconomic strata [7-9]. While vector control has generally been identified as being more equitable-with ITN distribution in particular identified as "pro-poor"-wealth inequities persist $[7,10]$ and Webster et al. [9] found that ever-treated net coverage was strongly biased towards richer households in almost all countries included in their study.

Vaccination is one of the most successful and costeffective public health measures, and has the potential to greatly reduce inequity, particularly in low- and middleincome countries [11,12]. The Expanded Programme on Immunization (EPI) has been a key catalyst in expanding access to childhood vaccines, with an estimated $86 \%$ coverage of DTP3 globally and 78\% coverage in Africa in 2018 [13]. However, gaps in coverage do remain, with higher levels of inequality in countries with lower vaccine uptake and evidence of "pro-rich" coverage in some countries $[14,15]$. Identifying those who are not receiving basic vaccines is therefore a priority $[16,17]$. The majority of children who remain unvaccinated are geographically concentrated, with $60 \%$ of these children residing in ten countries (including four malaria endemic countries in Africa: Angola, the Democratic Republic of Congo (DRC), Ethiopia, and Nigeria) [16].

The RTS,S/AS01 vaccine for Plasmodium falciparum malaria is the first vaccine to show partial protection against clinical and severe malaria in children. The multisite phase 3 trial of the RTS,S vaccine demonstrated $39.0 \%$ (95\% CI 34.3-43.3\%) protective efficacy against clinical malaria in young children who received all four doses, over 4 years of follow-up according to the perprotocol population [18]. Pilot implementation of the vaccine is now underway in three African countriesGhana, Kenya and Malawi [19] - and the findings from this cluster-randomized pilot study will inform public health policy decisions about wider roll-out of the vaccine, including the potential for RTS,S to be incorporated in the EPI [20].

This study sought to assess the potential of RTS,S to avert malaria cases, in malaria-endemic African countries, in light of the coverage of EPI vaccines compared to other malaria interventions. Using data from householdbased Demographic and Health Surveys (DHS), individual children were stratified into groups on the basis of reported ITN coverage (ownership and usage) and DTP3 vaccination status [21]. These groupings were used to explore socio-economic factors driving intervention uptake and to quantify the potential that introduction of the RTS,S vaccine could have in reducing malaria burden in those who are not currently accessing or using ITNs.

\section{Methods}

\section{Data sources}

Data were obtained from DHS and Malaria Indicator Surveys (MIS) in Africa [21]. Both are large, nationally representative, household surveys typically conducted every three to 4 years. The initial scope included all African countries with at least one administrative 1 (admin1) unit with $P$. falciparum prevalence in 2-10-year-old individuals $\left(P f \mathrm{PR}_{2-10}\right)$ greater than $10 \%$ based on Malaria Atlas Project (MAP) estimates for 2016 [22]. This threshold was chosen as the level above which the RTS,S malaria vaccine has been estimated to be highly costeffective [23]. Twenty-six countries met the inclusion criteria. For each, the most recent DHS and MIS, where geolocation data and parasite prevalence data were also available, were identified. Geolocation data were not available for South Sudan, Equatorial Guinea or Niger, and prevalence data were not available for Chad, Gabon or the Central African Republic. These countries were, 
therefore, excluded from the analysis. This resulted in 20 countries eligible for analysis with a median country-level parasite prevalence of $P f \mathrm{PR}_{2-10}=23 \%$ (range 5-43\%). The DHS and/or MIS dataset used for each country is listed in Additional file 1: Table S1.

To quantify vaccination uptake, individual-level data on the childhood vaccination status of DTP3 (administered at 14 weeks of age) and measles (administered at 9 months of age) for children aged 12-35 months were extracted from the DHS. A child was defined as having received DTP3 if they had received three doses, and having received the measles vaccine if they had received the first dose. This could be recorded by a mark on a vaccine card or a mother's response. DTP3 and measles vaccine coverage were found to be highly correlated at the country level (Additional file 1: Fig. S2) and, therefore, DTP3 vaccination status was used as an indicator of access to vaccination.

All DHS and MIS collect information on ITNs, therefore data on ITN access and usage could be linked to data on vaccination uptake at the individual level. A child was defined as using an ITN if they slept in the house on the previous night and used either an ITN, or both an ITN and untreated net, that previous night (these are both options in the DHS survey). A child was defined as having access to a net if they slept in the house on the previous night and had a mosquito bed net for sleeping. IRS was not considered in this analysis due to the low global percentage of households protected by this method.

Data on P. falciparum malaria prevalence and the proportion of children seeking treatment for fever were not consistently available in all DHS surveys and, therefore, could only be matched at the country or admin-1 level, using MIS data where possible (Additional file 1: Table S1). Treatment coverage was defined as the proportion of children aged between 12 and 59 months for whom fever was reported within the previous 2 weeks and who sought medical treatment. P. falciparum malaria prevalence was based on rapid diagnostic test (RDT) results in children aged between 12 and 59 months. The prevalence data were not included in the survey data sets for two countries at the individual level (Cameroon and Zambia), therefore, these values were obtained directly from the corresponding reports aggregated at the admin-1 level (Additional file 1: Table S1). All country and admin-1 coverage estimates were adjusted using the reported sample weights according to the DHS guidance [21].

Data on gender, age, rural/urban status, the highest level of the mother's education, and the wealth index were extracted to explore additional determinants underlying variation in access to vaccines and malaria interventions. These variables are included in the DHS and were available for the same children who had information recorded about vaccination status, ITN access and ITN usage. All data were extracted using the $r d h s$ package in $\mathrm{R}$ software [24].

\section{Analysis}

Six groups were defined, based on the vaccination status, ITN access and ITN usage for each child:

1. Did not receive the DTP3 vaccine and did not have access to or sleep under an ITN;

2. Did not receive the DTP3 vaccine and had access to, but did not sleep under, an ITN;

3. Did not receive the DTP3 vaccine but did sleep under an ITN;

4. Received the DTP3 vaccine but did not have access to or sleep under an ITN;

5. Received the DTP3 vaccine had access to, but did not sleep under, an ITN; and

6. Received the DTP3 vaccine and slept under an ITN.

The first group was termed "missed children" since these children do not have access to an ITN and are also unlikely to benefit from the introduction of the malaria vaccine introduced via the EPI. The fourth and fifth groups were termed "prospective children" since these children are not currently using ITNs but could be accessed via EPI to receive the malaria vaccine. The variation in associations between ITN usage and vaccine uptake were tested using the Breslow Day test (to assess conditional independence) and the Woolf test (to assess homogeneous associations) whilst accounting for correlation at country level by use of stratification.

A multinomial mixed effect model for nominal (unordered) outcomes was fitted to explore the determinants of overlap in vaccine coverage and ITN access and usage. This allowed groups $2-6$ to be contrasted against the reference group (the "missed children"), while accounting for variation at the country level using a random intercept $[25,26]$. The following variables were considered during the model building process: age of the child; malaria prevalence of the region in which the child lived; sex of the child; mother's education level; and whether the region in which a child lived was classified as rural or urban. Within each country, for each outcome level, there were an average of 2.24 observations associated with each survey cluster. Therefore, it was not feasible to consider clustering at the survey cluster level due to of the very few observations available. Prevalence of malaria parasitaemia was not found to be a significant variable and was subsequently removed from the model. These analyses were undertaken using SAS version 9.4 [27]. 
The number of children aged 12-59 months at risk of malaria for each country were estimated by multiplying the proportion of children in each group at the at the admin-1 level by the at-risk population for each admin-1 unit and aggregating it to the country level. Demographic and population data were obtained from the United Nations World Population Prospects 2015 and 2016 projections, respectively [28], and population spatial distributions from the GPW dataset [29]. Populations-at-risk were estimated as those living within the spatial limits of $P$. falciparum [30]. The number of cases that could be averted by the vaccine in prospective children were then estimated using only the at-risk populations of the prospective group who received the DTP3 vaccine but did not use an ITN (groups 4 and 5). The number of cases averted for those who already have a vaccine and use an ITN (group 6) were also calculated. For groups 4-6, it was assumed that all children who received DTP3 would also receive the RTS,S malaria vaccine (thereby estimating impact for a country-specific upper bound of coverage), and that the vaccine reduces the clinical incidence of malaria across this age group by $39 \%$ irrespective of whether they used an ITN [31]. The number of cases averted was calculated at the admin-1 level and aggregated at the country level. To calculate uncertainty ranges for cases averted, we applied the upper and lower bounds of the $95 \%$ confidence interval for vaccine efficacy reported in the clinical trial $(43.3 \%$ and $34.3 \%$ respectively [18]). To obtain the clinical incidence prior to vaccine introduction, a model-based relationship between parasite prevalence and clinical incidence (Additional file 1: Fig. S2) was used to translate the estimates of parasite prevalence by RDT in children younger than 5 years from the corresponding DHS or MIS [32].

\section{Results}

Figure 1 shows the country-level relationship between DTP3 vaccine coverage and three malaria intervention coverage indicators (ITN usage, ITN access and the proportion of fever cases seeking treatment). Across the majority of countries, vaccination coverage was higher than any of the selected malaria intervention coverage indicators. The highest levels of malaria intervention coverage had access to ITNs; with eight of the 20 countries analysed having higher ITN access compared to vaccination coverage (Angola, Benin, Côte d'Ivoire, DRC, Guinea, Mali, Nigeria and Uganda). However, this difference was only substantial (>10\%) in three of these countries (Benin, Malawi and Nigeria). Furthermore, there was a consistent gap between ITN access and ITN usage in all countries. ITN usage was lower than vaccination coverage in all but three countries (Benin, DRC and Mali). Only one country
(Angola) had lower vaccine coverage than proportion of children seeking treatment for fever.

Overall, 34\% of children did not use ITNs (groups 4 and 5: either no access or access but did not use) but received the DTP3 vaccine, and 35\% of children both used ITNs and received the DTP3 vaccine (Table 1). There was significant variation in the association between ITN use and DTP3 vaccine uptake between countries (Breslow Day and Woolf tests both had values $<0.001)$. The highest proportion of children who received no DTP3 vaccine and did not use an ITN were in south-western Africa, whereas the lowest proportion were in the south-east of Africa (Fig. 2A). In contrast, the highest proportion of children who received the DTP3 vaccine and did not use an ITN were in southeastern Africa and the lowest were south-western Africa (Fig. 2B). Examining unvaccinated children, 16\% of children used ITNs but were not vaccinated, and 15\% of children did not receive either intervention.

In the 20 countries analysed it was estimated that there are 33 million children at risk of malaria who currently do not use an ITN (Table 1). Of these, 23 million $(70 \%)$ are in the "prospective" group that did receive the DTP3 vaccine and hence could potentially receive protection from the malaria vaccine under existing distribution channels. Cameroon, DRC, Kenya, Mozambique, Nigeria, Tanzania and Uganda each have more than one million children in this group. However, Angola, DRC, and Nigeria each have more than one million children in the "missed children" category who did not receive the DTP3 vaccine and do not have access to a net. Across the 20 countries, approximately the same number of children in the "prospective" group (children who receive DTP3 but are not protected by an ITN), who do not currently use an ITN, had access to an ITN than did not despite individual countries having very different levels of overall access to ITNs. This pattern was repeated in the "missed children" group.

By vaccinating all children in the "prospective" group up to an estimated 9.7 million (uncertainty range 8.510.8 million) malaria cases could be averted each year across these 20 countries, assuming that all children who receive the DTP3 vaccine would receive all four doses of the RTS,S malaria vaccine (Table 2). An additional 10.8 million (9.5-12.0 million) cases could be averted by vaccinating those 23 million children who both use an ITN and have the DTP3 vaccine. Despite the relatively low vaccination coverage levels in Nigeria, $30 \%$ of total cases averted would be averted by vaccinating children in this country with a further $22 \%$ in two additional countries-Cameroon and the DRC.

The associations between demographic and socioeconomic variables and the different intervention 


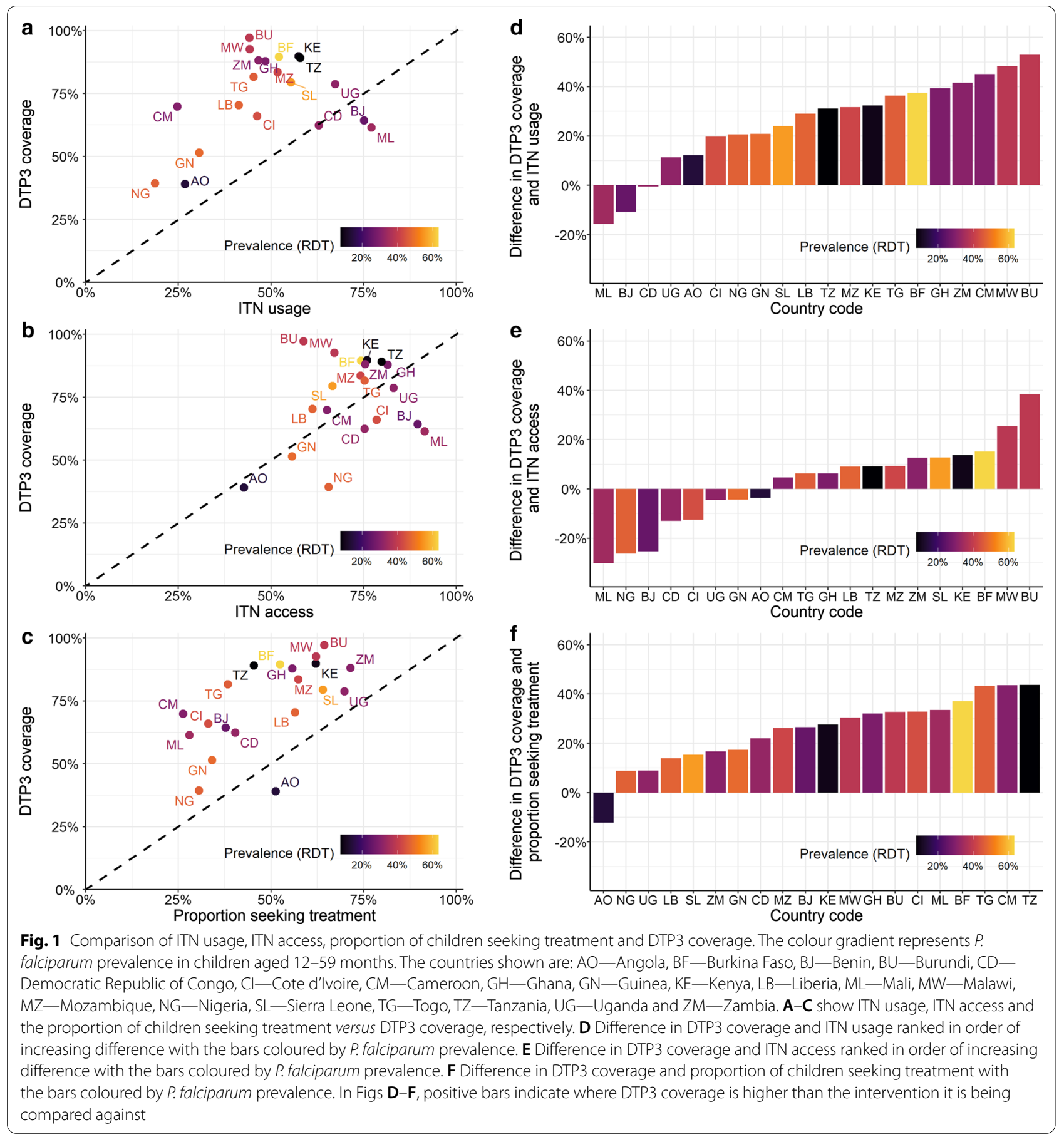

coverage groups are shown in Fig. 3. Older children (aged 24-35 months) were less likely to have received the DTP3 vaccine and use an ITN compared to younger children (aged 12-23 months). There were notable differences in intervention coverage between rural and urban populations; those that had access to or used a net were more likely to reside in rural areas compared to the missed children regardless of vaccination status $(\mathrm{p}=0.034)$ whereas those that received the DTP3 vaccine, but did not have access to a net, were more likely to reside in urban areas compared to the missed children $(\mathrm{p}=0.006)$. The mother's education status 
Table 1 Numbers of children aged 12-59 months at risk of malaria and receiving different intervention combinations (DTP3 vaccination and/or an ITN)

\begin{tabular}{|c|c|c|c|c|c|}
\hline Country & $\begin{array}{l}\text { Total number of } \\
\text { children, thousands }\end{array}$ & $\begin{array}{l}\text { Children without ITN } \\
\text { or vaccine, thousands }\end{array}$ & $\begin{array}{l}\text { Children without ITN but } \\
\text { vaccinated, thousands }\end{array}$ & $\begin{array}{l}\text { Children with ITN but } \\
\text { no vaccine, thousands }\end{array}$ & $\begin{array}{l}\text { Children with ITN and } \\
\text { vaccine, thousands }\end{array}$ \\
\hline Angola & 2672 & $1243(47 \%)$ & 715 (27\%) & $402(15 \%)$ & $312(12 \%)$ \\
\hline Benin & 1043 & 104 (10\%) & $153(15 \%)$ & $272(26 \%)$ & $513(49 \%)$ \\
\hline Burkina Faso & 1901 & $116(6 \%)$ & 797 (42\%) & $76(4 \%)$ & $912(48 \%)$ \\
\hline Burundi & 1097 & $20(2 \%)$ & $602(55 \%)$ & $12(1 \%)$ & $463(42 \%)$ \\
\hline Cameroon & 4361 & 904 (21\%) & 2753 (63\%) & $129(3 \%)$ & $575(13 \%)$ \\
\hline $\begin{array}{l}\text { Democratic } \\
\text { Republic of } \\
\text { Congo }\end{array}$ & 8252 & $1522(18 \%)$ & 1842 (22\%) & $1829(22 \%)$ & 3060 (37\%) \\
\hline Côte d'Ivoire & 2132 & 399 (19\%) & $776(36 \%)$ & $279(13 \%)$ & 677 (32\%) \\
\hline Ghana & 2242 & 167 (7\%) & $967(43 \%)$ & $103(5 \%)$ & 1006 (45\%) \\
\hline Guinea & 1453 & $643(44 \%)$ & $373(26 \%)$ & $220(15 \%)$ & $217(15 \%)$ \\
\hline Kenya & 3365 & 167 (5\%) & $1122(33 \%)$ & $148(4 \%)$ & 1928 (57\%) \\
\hline Liberia & 406 & $71(18 \%)$ & $165(41 \%)$ & $49(12 \%)$ & $121(30 \%)$ \\
\hline Malawi & 1752 & $82(5 \%)$ & $893(51 \%)$ & $46(3 \%)$ & 730 (42\%) \\
\hline Mali & 1967 & $220(11 \%)$ & $243(12 \%)$ & $580(29 \%)$ & $923(47 \%)$ \\
\hline Mozambique & 2813 & $288(10 \%)$ & 1041 (37\%) & 202 (7\%) & 1281 (46\%) \\
\hline Nigeria & 18,589 & 3281 (18\%) & $5758(31 \%)$ & 5005 (27\%) & 4545 (24\%) \\
\hline Sierra Leone & 573 & $61(11 \%)$ & $201(35 \%)$ & $58(10 \%)$ & $253(44 \%)$ \\
\hline Tanzania & 5348 & $218(4 \%)$ & $2143(40 \%)$ & $313(6 \%)$ & 2674 (50\%) \\
\hline Togo & 964 & $122(13 \%)$ & $426(44 \%)$ & $58(6 \%)$ & $358(37 \%)$ \\
\hline Uganda & 4189 & $351(8 \%)$ & 1027 (25\%) & $497(12 \%)$ & $2314(55 \%)$ \\
\hline Zambia & 1701 & $114(7 \%)$ & $803(47 \%)$ & $83(5 \%)$ & $702(41 \%)$ \\
\hline Total & 66,821 & $10,094(15 \%)$ & 22,801 (34\%) & $10,363(16 \%)$ & $23,564(35 \%)$ \\
\hline
\end{tabular}

The percentage is relative to the total for that country. The analysis was performed at the admin-1 unit level and the total numbers of children were then aggregated at the country level. A child without an ITN either had no access to an ITN or had access but did not use it

was strongly associated with intervention uptake with membership of all intervention groups that either used an ITN and/or received the DTP3 vaccine (groups 3, 4,5 and 6 ) being significantly associated with higher levels of education $(\mathrm{p}<0.001)$. Furthermore, membership of the group that did not receive the vaccine and had access to a net but did not use it (group 2) was significantly less likely in those that had received higher education compared to those that received little or no education. The wealth index was also a strong predictor of intervention coverage. Membership of all intervention groups that had either received the DTP3 vaccine, or owned and/or used a net, was significantly associated with higher wealth levels after controlling for the urban/rural divide. Those receiving higher incomes were approximately twice as likely to access both nets and vaccines (groups 5 and 6) compared to those in the lower wealth quintile $(p<0.001)$. The numbers of children in each group are provided in Additional file 1: Table S2.

\section{Discussion}

The substantial declines in the burden of malaria in subSaharan Africa since 2010 have been attributed primarily to the rapid increase in access and usage of ITNs alongside slower but significant improvements in access to first-line treatment [33]. However, in more recent years the coverage of both interventions has plateaued, with the most recent household surveys analysed here demonstrating sub-optimal levels of ITN usage in many countries (at or below 50\%) and even lower rates of treatment seeking for fever. In contrast, supported by the establishment of Gavi, The Vaccine Alliance in 2000 and the WHO's EPI, uptake of childhood vaccination has steadily increased, although the past decade has seen some stagnation [16, 34]. As demonstrated in this analysis, vaccine coverage is high in most of the 20 countries studied here, with only two countries (Angola and Guinea) reporting under $50 \%$ uptake in their most recent DHS. Furthermore, these results demonstrate that coverage of vaccines administered via the EPI is substantially higher 

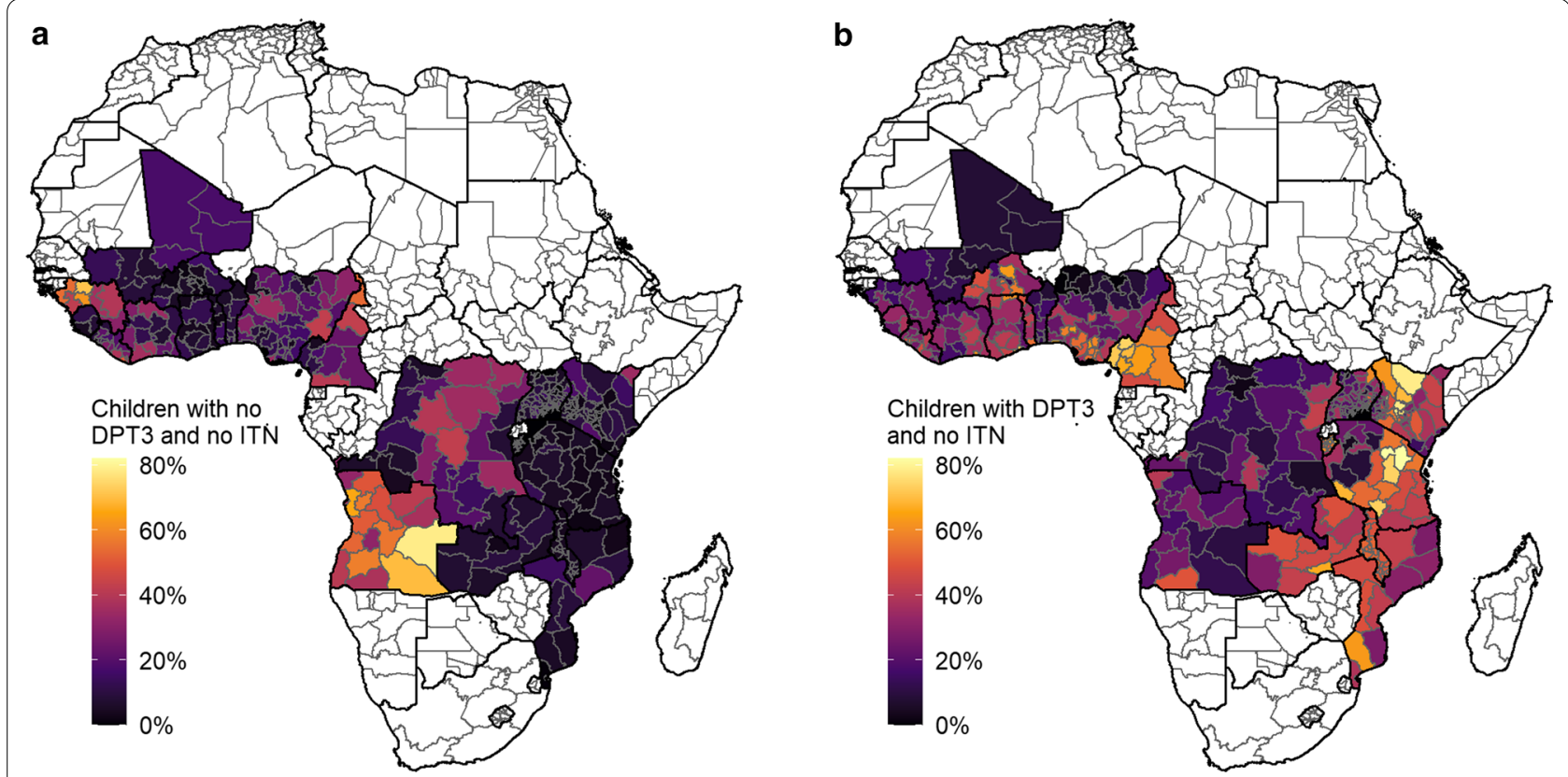

Fig. 2 Proportion of the population aged 12-59 months in each country who do not use an ITN (includes both access and does not use, and no access) and by vaccination status. A Proportion of children who have not been vaccinated with the DTP3 vaccine and did not use an ITN. B Proportion of children who have been vaccinated with the DTP3 vaccine and did not use an ITN. The countries shown are Angola, Burkina Faso, Benin, Burundi, Democratic Republic of Congo, Cote d'Ivoire, Cameroon, Ghana, Guinea, Kenya, Liberia, Mali, Malawi, Mozambique, Nigeria, Sierra Leone, Togo, Tanzania, Uganda and Zambia

than usage of ITNs or fever treatment-seeking rates in the majority of countries, which is consistent with other research [9]. This creates an opportunity to consider roll-out strategies for the introduction of a malaria vaccine that are distinct to implementation programmes for other malaria interventions, to maximize impact by building on the wider reach of the Expanded Programme on Immunization.

As noted elsewhere, the utilization of malaria interventions and uptake of vaccination were found to be strongly associated with demographic and socioeconomic indicators [7-10, 14, 15]. Perhaps not surprisingly given higher malaria prevalence, those accessing and using ITNs were more likely to reside in rural areas, whereas as higher uptake of the DTP3 vaccine was associated with urban areas, possibly because of better access to healthcare facilities. Uptake of vaccination was also strongly associated with both the mother's educational level and the wealth quintile, although as noted elsewhere, even at lower levels of education and wealth, the coverage of vaccination remained high [15]. Access to ITNs and ITN usage were also both strongly associated with the mother's education status and wealth; however, having access to a net but not using it whilst also not being vaccinated with DTP3 was more strongly associated with lower educational status of mothers than with wealth. In addition, vaccine and ITN usage were found to be associated with age. Vaccine coverage may have improved over time so older children were less likely to be vaccinated than younger children and some net campaigns such as through antenatal care may better target younger children.

It was estimated that there are currently 33 million children in these 20 countries who are not using an ITN. Of these, 23 million (70\% of the total children without an ITN) are estimated to have received the DTP3 vaccine and hence could be reached by the EPI. If the RTS,S malaria vaccine were made available to just these 23 million children, up to an estimated 9.7 million cases (or 0.44 cases per vaccinated child) could be averted each year (assuming all children who receive the DTP3 vaccine also receive the RTS,S). If the vaccine was also administered to those children with an ITN and who were vaccinated ( 24 million additional children), up to an estimated additional 10.8 million cases (or 0.47 cases per vaccinated child) could also be averted. Around $40 \%$ of the total cases would be averted in the Democratic Republic of Congo and Nigeria, countries which are currently contributing large proportions of malaria cases worldwide. This alone could represent a substantial reduction in the global malaria burden, reducing $P$. falciparum malaria cases by approximately $4 \%$. However, there are still 9.8 
Table 2 Estimated malaria cases averted

\begin{tabular}{|c|c|c|c|c|c|}
\hline \multirow[t]{2}{*}{ Country } & \multirow{2}{*}{$\begin{array}{l}\text { Total cases averted in } \\
\text { thousands }\end{array}$} & \multicolumn{2}{|l|}{ Children using an ITN } & \multicolumn{2}{|l|}{ Children not using an ITN } \\
\hline & & $\begin{array}{l}\text { Cases averted in } \\
\text { thousands (uncertainty } \\
\text { range) }\end{array}$ & $\begin{array}{l}\text { Percentage of total } \\
\text { averted across all } \\
\text { countries }\end{array}$ & $\begin{array}{l}\text { Cases averted in } \\
\text { thousands (uncertainty } \\
\text { range) }\end{array}$ & $\begin{array}{l}\text { Percentage of total } \\
\text { averted across all } \\
\text { countries }\end{array}$ \\
\hline Angola & $162(143-180)$ & $54(47-60)$ & 0 & $109(96-121)$ & 1 \\
\hline Benin & $282(248-313)$ & $220(193-244)$ & 1 & $63(55-69)$ & 0 \\
\hline Burkina Faso & $1326(1167-1473)$ & $729(641-809)$ & 4 & $597(525-663)$ & 3 \\
\hline Burundi & $583(513-647)$ & $237(208-263)$ & 1 & $346(305-385)$ & 2 \\
\hline Cameroon & 1359 (1195-1508) & $219(193-243)$ & 1 & $1140(1002-1265)$ & 6 \\
\hline $\begin{array}{l}\text { Democratic } \\
\text { Republic of } \\
\text { Congo }\end{array}$ & 2307 (2029-2562) & $1420(1249-1576)$ & 7 & $888(781-985)$ & 4 \\
\hline Côte d'Ivoire & $834(733-926)$ & $433(381-481)$ & 2 & $400(352-445)$ & 2 \\
\hline Ghana & $836(736-929)$ & $465(409-516)$ & 2 & $371(327-412)$ & 2 \\
\hline Guinea & $396(348-439)$ & $160(140-177)$ & 1 & $236(208-262)$ & 1 \\
\hline Kenya & $536(471-595)$ & $399(351-443)$ & 2 & $137(120-152)$ & 1 \\
\hline Liberia & $222(195-246)$ & $94(82-104)$ & 0 & $128(113-142)$ & 1 \\
\hline Malawi & $930(818-1033)$ & $416(366-462)$ & 2 & $514(452-571)$ & 3 \\
\hline Mali & $575(506-638)$ & $465(409-516)$ & 2 & $110(97-123)$ & 1 \\
\hline Mozambique & 1251 (1100-1389) & $692(608-768)$ & 3 & $559(492-621)$ & 3 \\
\hline Nigeria & 5684 (4999-6311) & 2887 (2539-3205) & 14 & $2797(2460-3106)$ & 14 \\
\hline Sierra Leone & $351(309-390)$ & $204(180-227)$ & 1 & $147(129-163)$ & 1 \\
\hline Tanzania & $522(459-580)$ & $338(297-376)$ & 2 & $184(162-204)$ & 1 \\
\hline Togo & $578(508-641)$ & $264(232-293)$ & 1 & $314(276-348)$ & 2 \\
\hline Uganda & 1317 (1158-1462) & 889 (782-987) & 4 & $428(377-476)$ & 2 \\
\hline Zambia & $491(432-545)$ & $245(215-272)$ & 1 & $246(217-273)$ & 1 \\
\hline Total & $20,542(18,066-22,807)$ & $10,827(9522-12,021)$ & 53 & $9715(8544-10,786)$ & 47 \\
\hline
\end{tabular}

The numbers of cases, and percentage relative to the total across all countries, that could be averted annually in 12-59-month-old children if universal coverage of the RTS,S/AS01 vaccine was achieved, for those who received the DTP3 vaccine in each country, assuming that all children who received the DTP3 vaccine also received all four doses of the RTS,S malaria vaccine. These estimates are calculated for individual admin-1 units and aggregated at the country level. Uncertainty ranges in parentheses represent the calculated impact based on the $95 \%$ vaccine efficacy confidence interval reported in the clinical trial [18]

million children across the countries considered who would remain unprotected by either intervention; these "missed children" should remain the focus of initiatives to improve equity in access to both malaria interventions and vaccination.

There are several limitations to this analysis. First, the analysis was only undertaken for 20 of the 27 malariaendemic countries of interest within sub-Saharan Africa. Those countries for which data were not available contributed around 10\% of the Africa malaria burden in 2018 and, therefore, also remain an important target for both malaria interventions and vaccination [6]. Second, due to the different survey designs, vaccination status and access to treatment were not able to be linked at the individual level. Given that both rely on access to health services, it is likely that these may be correlated, although levels of access to treatment remain well below vaccination rates. An alternative equity dimension that may be relevant to consider when targeting malaria interventions could be the potential for access to rapid treatment since both severe disease incidence and malaria mortality have been associated with the time taken to reach care $[35,36]$. The definition of treatment used for this analysis is also limited because, depending on the policy within each country, in addition to presenting with a fever, a child would generally need to test positive for malaria using a rapid diagnostic test before being administered treatment. In addition, some health centres may occasionally experience treatment shortages. Third, to estimate the impact of the RTS,S vaccine on malaria burden the mean estimate of vaccine efficacy across the phase 3 trial sites over a 4-year period (39\%) was applied. As usage of ITNs during the trial was very high, this likely represents an under-estimate of the true impact of the vaccine in this group of prospective children. A study using RTS,S trial and bed net usage in Malawi estimated that vaccinating a child in urban Lilongwe without a bed net could prevent 1.09 malaria cases, versus 0.67 for a child with a bed net. In rural Lilongwe, 2.59 and 1.59 malaria cases could be averted, respectively [37]. Furthermore, in 


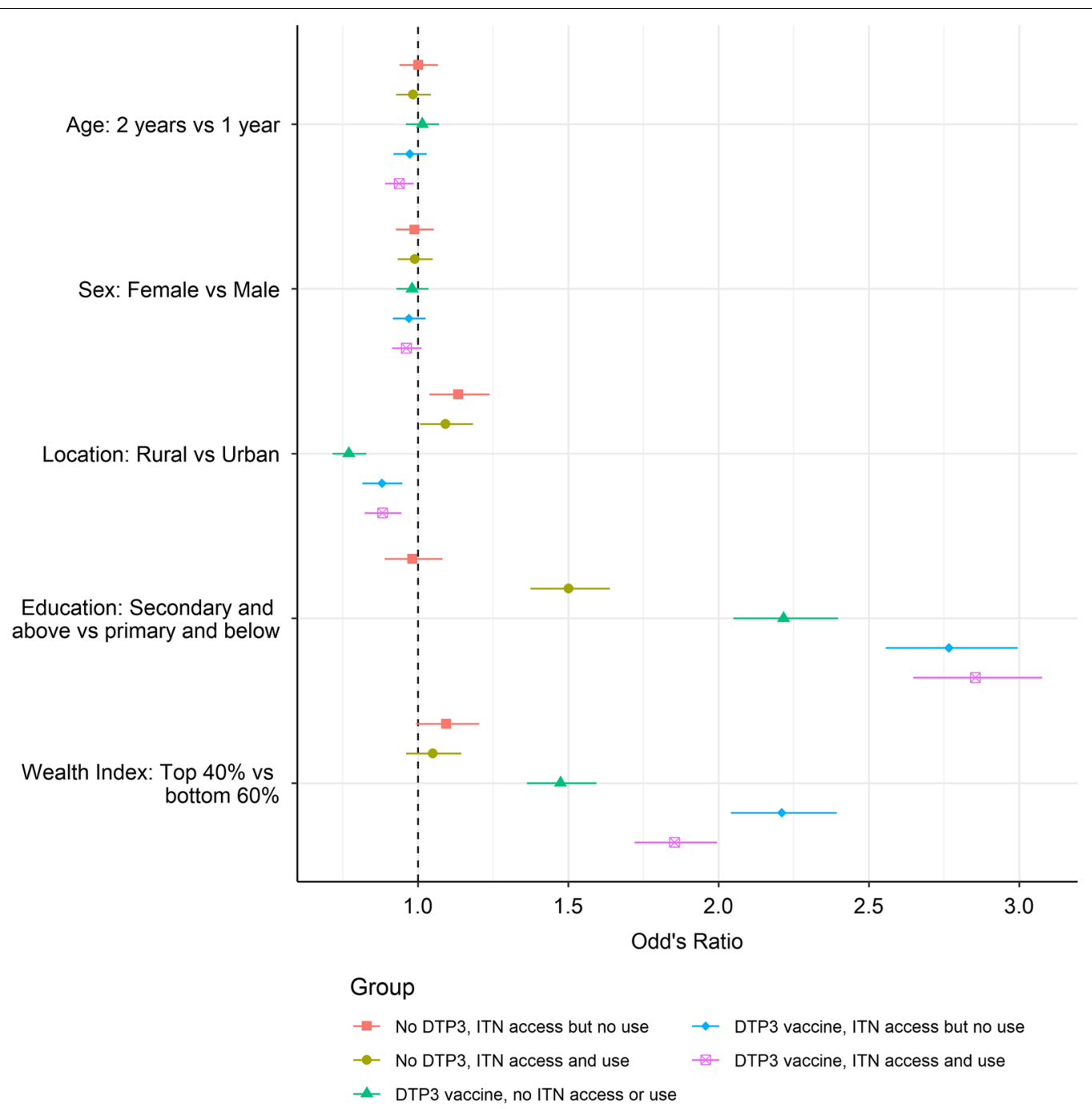

Fig. 3 The relationship between demographic and socioeconomic variables and intervention coverage. Odds ratio estimates, 95\% confidence levels and $p$ values are shown for each of the predictors. All ratios are comparative to group 1 where children did not receive the DTP3 vaccine and have no access to an ITN (the "missed children"). Children in group 2 did not receive the DTP3 vaccine and had access to, but did not sleep under, an ITN; children in group 3 did not receive the DTP3 vaccine but did sleep under an ITN; children in group 4 received the DTP3 vaccine but did not have access to or sleep under an ITN; children in group 5 received the DTP3 vaccine and had access to, but did not sleep under, an ITN; and children in group 6 received the DTP3 vaccine and slept under an ITN

taking this simple approach to estimating vaccine impact, the differences in vaccine efficacy by endemicity that were observed in the trial, and the potential age-shifting of cases that would likely occur as a result of reduced exposure to infection, were not considered [23]. Fourth, universal malaria vaccine coverage for children who receive the DTP3 vaccine was assumed, which results in the estimate of cases averted being an upper bound. The RTS,S vaccine is delivered as a four-dose schedule, with the first and third doses aligning with existing EPI contact points. Therefore, particularly in the early phase of vaccine introduction, it is possible that vaccine coverage could be lower than that of DTP3. In addition, coverage of the fourth dose is expected to be lower given the reduced health system contact as children become older. Some protection is still conferred from the first three doses (as demonstrated in the phase 3 trial), however the present analysis was not designed to estimate the impact of multiple levels of efficacies. Projected vaccine coverage will be informed by data from the pilot implementation studies as they progress. Fifth, this analysis was based on self-reported vaccination status and ITN use from household surveys across different years, which may not reflect the current situation; as such they are not directly 
comparable with estimates produced by the WHO (for malaria interventions and vaccination) and UNICEF (for vaccination), which are obtained by triangulating data from a number of sources. Several studies show that selfreporting vaccination often leads to a slight overestimate of true coverage $[38,39]$. Finally, this analysis scaled up restricted surveys on intervention coverage in order to obtain regional/national estimates of malaria cases that would be prevented if RTS,S was widely deployed. While this extrapolation of survey data introduces considerable uncertainty, these estimates still provide an indication of wider future RTS,S impact.

\section{Conclusions}

In summary, broadly high levels of childhood vaccination across malaria-endemic countries in sub-Saharan Africa, including in high P. falciparum prevalence regions where take-up of current interventions remains sub-optimal, provide an opportunity to maximize the impact of childhood malaria vaccination. Older children were less likely to have received the DTP3 vaccine and use an ITN compared to younger children and the higher the levels of mother's education and wealth indexes, the greater the intervention coverage. This study also highlights the considerable number of children who are not currently accessing routine childhood immunizations or core malaria interventions and who should be the focus of health equity initiatives in order to improve access. These combined findings allow for the identification of populations that could benefit most from the introduction of a childhood malaria vaccine and could be used to devise strategies for future malaria vaccine implementation.

\section{Abbreviations}

WHO: World Health Organization; GTS: World Health Organization Global Technical Strategy for Malaria; ITN: Insecticide-treated net; DTP3: Diphtheriatetanus-pertussis vaccine dose 3; IRS: Indoor residual spraying; RTS,S: RTS,S/ AS01 malaria vaccine for children; DHS: Demographic and Health Surveys; MIS: Malaria Indicator Surveys; EPI: Expanded Programme on Immunization; MAP: Malaria Atlas Project; $P \mathrm{PPR}_{2-10}$ : P. falciparum Parasite prevalence in 2-10-yearold individuals; IQR: Inter-quartile range; DRC: Democratic Republic of Congo.

\section{Supplementary Information}

The online version contains supplementary material available at https://doi. org/10.1186/s12936-021-03966-x.

Additional file 1. Analysis of the potential for a malaria vaccine to reduce gaps in malaria intervention coverage.

\section{Acknowledgements}

Not applicable.

\section{Authors' contributions}

ABH and ACG conceived and designed the study. HJTU, ABH and LM extracted the data and ran the analyses. All authors interpreted the findings. HJTU and
$\mathrm{ABH}$ wrote the first draft of the paper. All authors read and approved the final manuscript.

\section{Funding}

This work was funded by Grants from PATH (ABH, ACG) and the Bill and Melinda Gates Foundation (HJTU, LM, ACG). HJTU, PW and ABH acknowledge support from their Imperial College Research Fellowships. All authors acknowledge funding from the MRC Centre for Global Infectious Disease Analysis (MR/ R015600/1), jointly funded by the UK Medical Research Council (MRC) and the UK Foreign, Commonwealth \& Development Office (FCDO), under the MRC/FCDO Concordat agreement and is also part of the EDCTP2 programme supported by the European Union. LM currently receives funding from the National Institute for Health Research (NIHR) Community Healthcare MedTech and In Vitro Diagnostics Co-operative at Oxford Health NHS Foundation. PATH contributed to the interpretation of results and provided comments on the manuscript. All other funders of the study had no role in study design, data analysis, interpretation of findings, or drafting of the manuscript.

\section{Availability of data and materials}

The data that support the findings of this study are available from the DHS Program repository (https://dhsprogram.com/) and can be accessed upon registration and application.

\section{Declarations}

Ethics approval and consent to participate

Not applicable.

\section{Consent for publication}

Not applicable.

\section{Competing interests}

The authors declare that they have no competing interests.

\section{Author details}

${ }^{1}$ MRC Centre for Global Infectious Disease Analysis, School of Public Health, Imperial College London, St Mary's Campus, Norfolk Place, London W2 1PG, UK. ${ }^{2}$ Nuffield Department of Primary Care Health Sciences, University of Oxford Radcliffe Observatory Quarter, Woodstock Road, Oxford OX2 6GG, UK.

Received: 9 October 2020 Accepted: 27 October 2021

Published online: 17 November 2021
References

1. Bhatt S, Weiss DJ, Cameron E, Bisanzio D, Mappin B, Dalrymple U, et al. The effect of malaria control on Plasmodium falciparum in Africa between 2000 and 2015. Nature. 2015;526:207-11.

2. WHO. World malaria report 2020. Geneva, World Health Organization, 2020. https://www.who.int/teams/global-malaria-programme/reports/ world-malaria-report-2020. Accessed 18 Jul 2019.

3. WHO. Global technical strategy for malaria, 2016-2030. Geneva, World Health Organization, 2015. https://www.who.int/malaria/publications/ atoz/9789241564991/en/. Accessed 18 Jul 2019.

4. WHO. High burden to high impact: a targeted malaria response. Geneva: World Health Organization; 2018.

5. WHO. World malaria report 2017. Geneva: World Health Organization; 2017.

6. WHO. World malaria report 2019. Geneva: World Health Organization; 2019.

7. Galactionova K, Tediosi F, Camponovo F, Smith TA, Gething PW, Penny MA. Country specific predictions of the cost-effectiveness of malaria vaccine RTS, S/AS01 in endemic Africa. Vaccine. 2017;35:53-60.

8. Chao F, You D, Pedersen J, Hug L, Alkema L. Articles National and regional under-5 mortality rate by economic status for low-income and middle-income countries : a systematic assessment. Lancet Glob Health. 2018;6:e535-47. 
9. Webster J, Lines J, Bruce J, Armstrong-Schellenberg J, Hanson K. Which delivery systems reach the poor? Equity of treated nets, untreated nets and immunisation to reduce child mortality in Africa. Lancet Infect Dis. 2005;5:709-17.

10. Li Z, Li M, Subramanian SV, Lu C. Assessing levels and trends of child health inequality in 88 developing countries: from 2000 to 2014. Glob Health Action. 2017;10:1408385.

11. Andre FE, Booy R, Bock HL, Clemens J, Datta SK, John TJ, et al. Vaccination greatly reduces disease, disability, death and inequity worldwide. Bull World Health Organ. 2008;86:140-6.

12. Greenwood B. The contribution of vaccination to global health: past, present and future. Philos Trans R Soc B. 2014;369:20130433.

13. Gavi The Vaccine Alliance. Gavi in Africa since 2000. Geneva. 2020. https:// www.gavi.org. Accessed 18 Jul 2019.

14. Hosseinpoor AR, Bergen N, Schlotheuber A, Gacic-Dobo M, Hansen PM, Senouci K, et al. State of inequality in diphtheria-tetanus-pertussis immunisation coverage in low-income and middle-income countries: a multicountry study of household health surveys. Lancet Glob Health. 2016;4:e617-26.

15. Arsenault C, Johri M, Nandi A, Mendoza JM, Hansen PM, Harper S. Country-level predictors of vaccination coverage and inequalities in Gavisupported countries. Vaccine. 2017;35:2479-88.

16. Piot $\mathrm{P}$, Larson HJ, Brien KLO, John N, Ng E, Sow S, et al. Immunization: vital progress, unfinished agenda. Nature. 2019;575:119-29.

17. WHO. Malaria vaccine: WHO position paper-January 2016. Wkly Epidemiol Rec. 2016:4:33-52.

18. RTSS Clinical Trials Partnership. Efficacy and safety of RTS,S/AS01 malaria vaccine with or without a booster dose in infants and children in Africa: final results of a phase 3 , individually randomised, controlled trial. Lancet. 2015;386:31-45.

19. Adepoju P. RTS,S malaria vaccine pilots in three African countries. Lancet. 2019;393:1685.

20. Hamel MJ. Malaria vaccine implementation programme (MVIP) update and framework for policy decision. 2018.

21. ICF. Data. The DHS program website. Funded by USAID. https://dhspr ogram.com. Accessed 18 Jul 2019

22. Weiss DJ, Lucas TCD, Nguyen M, Nandi AK, Bisanzio D, Battle KE, et al. Mapping the global prevalence, incidence, and mortality of Plasmodium falciparum, 2000-17: a spatial and temporal modelling study. Lancet. 2019;394:322-411.

23. Penny MA, Verity R, Bever CA, Sauboin C, Galactionova K, Flasche S, et al. Public health impact and cost-effectiveness of the RTS,S/AS01 malaria vaccine: a systematic comparison of predictions from four mathematical models. Lancet. 2016;387:367-75.

24. Watson OJ, FitzJohn R, Eaton JW. rdhs: an R package to interact with The Demographic and Health Surveys (DHS) Program datasets. Wellcome Open Res. 2019;4:103.
25. Hedeker D. A mixed-effects multinomial logistic regression model. Stat Med. 2003;22:1433-46.

26. Hartzel J, Agresti A, Caffo B. Multinomial logit random effects models. Stat Modelling. 2001;1:81-102.

27. SAS Institute Inc. SAS 9.4 for windows.

28. United Nations. World population prospects - population divisionUnited Nations. 2019. https://population.un.org/wpp/Download/Stand ard/Population/. Accessed 17 Jul 2019.

29. CIESIN. Gridded population of the world (GPW), v4|SEDAC. https://beta sedac.ciesin.columbia.edu/data/collection/gpw-v4. Accessed 4 May 2021.

30. Pfeffer DA, Lucas TCD, May D, Harris J, Rozier J, Twohig KA, et al. MalariaAtlas: an R interface to global malariometric data hosted by the Malaria Atlas Project. Malar J. 2018;17:352.

31. $\mathrm{WHO}$. Q\&A on the malaria vaccine implementation programme (MVIP). Geneva, World Health Organization. 2019. https://www.who.int/malaria/ media/malaria-vaccine-implementation-qa/en/. Accessed 17 Jul 2019.

32. Griffin JT, Hollingsworth TD, Okell LC, Churcher TS, White M, Hinsley W, et al. Reducing Plasmodium falciparum malaria transmission in Africa: a model-based evaluation of intervention strategies. PLoS Med. 2010;7: e1000324.

33. WHO. World Malaria Report 2018. Geneva, World Health Organization, 2018. https://www.who.int/malaria/publications/world-malaria-report2018/en/. Accessed 18 Jul 2019.

34. Gavi The Vaccine Alliance. Facts and figures. 2019. https://www.gavi.org/ programmes-impact/our-impact/facts-and-figures. Accessed 18 Jul 2019.

35. Meerman L, Ord R, Bousema JT, van Niekerk M, Osman E, Hallett R, et al. Carriage of chloroquine-resistant parasites and delay of effective treatment increase the risk of severe malaria in Gambian children. J Infect Dis. 2005;192:1651-7.

36. Mpimbaza A, Nayiga S, Ndeezi G, Rosenthal PJ, Karamagi C, Katahoire A. Understanding the context of delays in seeking appropriate care for children with symptoms of severe malaria in Uganda. PLOS ONE. 2019;14: e0217262.

37. Bell GJ, Loop M, Topazian HM, Hudgens M, Mvalo T, Juliano JJ, et al. Case reduction and cost-effectiveness of the RTS, S/AS01 malaria vaccine alongside bed nets in Lilongwe, Malawi. Vaccine. 2020;38:4079-87.

38. Binyaruka P, Borghi J. Validity of parental recalls to estimate vaccination coverage: evidence from Tanzania. BMC Health Serv Res. 2018;18:440.

39. Miles M, Ryman TK, Dietz $V$, Zell E, Luman ET. Validity of vaccination cards and parental recall to estimate vaccination coverage: a systematic review of the literature. Vaccine. 2013;31:1560-8.

\section{Publisher's Note}

Springer Nature remains neutral with regard to jurisdictional claims in published maps and institutional affiliations.
Ready to submit your research? Choose BMC and benefit from:

- fast, convenient online submission

- thorough peer review by experienced researchers in your field

- rapid publication on acceptance

- support for research data, including large and complex data types

- gold Open Access which fosters wider collaboration and increased citations

- maximum visibility for your research: over $100 \mathrm{M}$ website views per year

At BMC, research is always in progress.

Learn more biomedcentral.com/submissions 